\title{
Finding a homeomorphism between almost homeomorphic manifolds
}

\author{
By Kazuaki KoBAyashi
}

\section{§1. Introduction}

Throughout this paper we shall only be concerned with the piecewise linear category of polyhedra and piecewise linear maps. In this paper we investigate the following problem; Let $W_{1}$ and $W_{2}$ be two $P L$ manifolds whose interiors and boundaries are $P L$ homeomorphic each other. When are $W_{1}$ and $W_{2} P L$ homeomorphic?

We obtain the result that such homeomorphism problem is closely related to the $h$-cobordism near the boundary (see THEOREM 2).

$\partial M$ and Int $M$ stand for the boundary and the interior of the manifold $M$. $\cong$ means $P L$ homeomorphic. $I=[0,1]$ is a closed unit interval. $\# X$ means the order of a set $X$.

\section{$\S 2$.}

Definition 1. Let $W_{i}(i=1,2)$ be bounded manifolds. When $\partial W_{1} \cong$ $\partial W_{2}$ and Int $W_{1} \cong \operatorname{Int} W_{2}$, we say $W_{1}$ is almost homeomorphic to $W_{2}$. And we define $\mathscr{A}(W)=$ set of $P L$ homeomorphism classes of $P L$ manifolds which are almost homeomorphic to $W$.

Proposition 1. ([2. Th. 2, 4]) Let $W_{j}^{n}(j=1,2)$ be compact bounded $n$-manifolds $(n \geqq 6)$. Then Int $W_{1}^{n} \cong I n t W_{2}^{n}$ if and only if $W_{1}$ and $W_{2}$ are boundary h-cobordant i.e. there are h-cobordisms $\left(U^{(i)} ; \partial W_{2}^{(i)}, M^{(i)}\right)$ such that
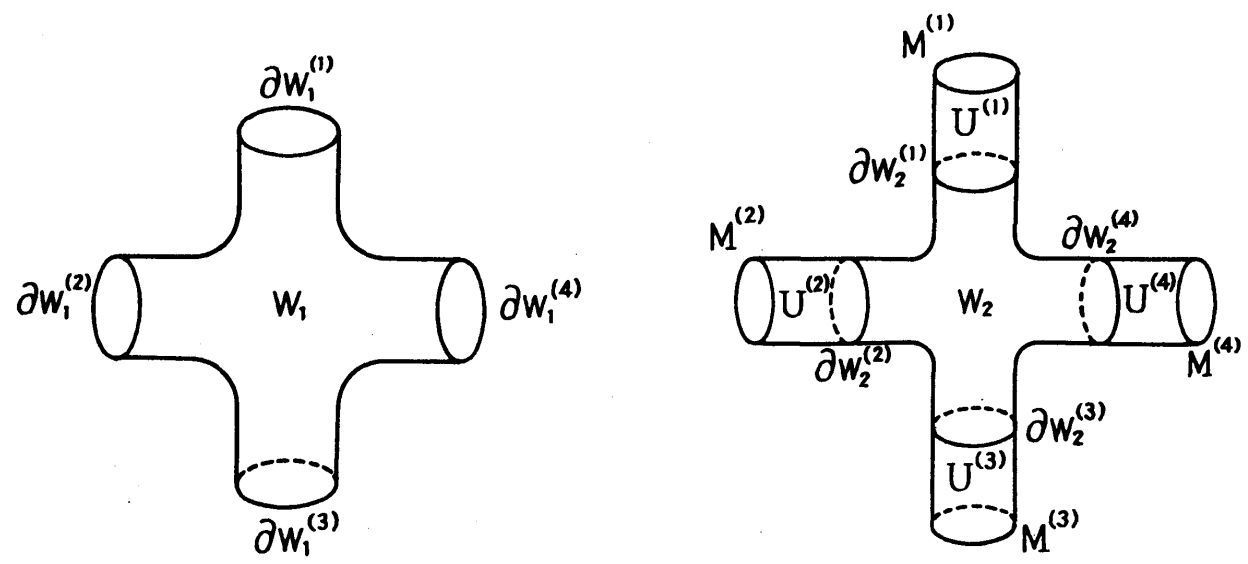

Fig. 1 . 


$$
\left(W_{1}, \partial W_{2}\right) \cong\left(W_{2} \cup_{\partial W_{2}^{(1)}}^{\cup} U^{(1)} \cup \cdots \underset{\partial W_{2}^{(p)}}{\cup} U^{(p)}, M^{(1)} \cup \cdots \cup M^{(p)}\right)
$$

where $p$ is the number of the components of $\partial W_{i}$.

LEMMA 1. Let $W_{i}(i=1,2)$ be compact bounded n-manifolds such that Int $W_{1} \cong$ Int $W_{2}$ and $g:$ Int $W_{1} \rightarrow$ Int $W_{2}$ be a given PL homeomorphism. Let $c_{1}: \partial W_{1} \times I \rightarrow W_{1}$ be an PL embedding such that $c_{1}(x, 0)=x$ for $x \in \partial W_{1}$ i.e. $c_{1}$ is a boundary collar and let $U_{g}$ be a region bounded by $\partial W_{2}$ and $g c_{1}\left(\partial W_{1} \times\{1\}\right)$ in $W_{2}$. If $U_{g} \cong \partial W_{1} \times I$, there is a PL homeomorphism $h$ : $W_{1} \rightarrow W_{2}$ such that

$$
h\left|W_{1}-c_{1}\left(\partial W_{1} \times[0,1]\right)=g\right| W_{1}-c_{1}\left(\partial W_{1} \times[0,1]\right)
$$

Proof. Let $\phi: \partial W_{1} \times I \rightarrow U_{g}$ be a $P L$ homeomorphism such that $\phi\left(\partial W_{1}\right.$ $\times\{0\})=\partial W_{2}$ and $\phi\left(\partial W_{1} \times\{1\}\right)=g c_{1}\left(\partial W_{1} \times\{1\}\right)$. Then we may define a $P L$ homeomorphism $h: W_{1} \rightarrow W_{2}$ by

$$
h(x)=\left\{\begin{array}{lll}
g(x) & \text { on } & x \in W_{1}-c_{1}\left(\partial W_{1} \times[0,1]\right) \\
\phi(u, \alpha) & \text { on } & x \in c_{1}\left(\partial W_{1} \times[0,1]\right)
\end{array}\right.
$$

where $u$ and $\alpha$ are decided as follows; since $x \in c_{1}\left(\partial W_{1} \times[0,1]\right)$, it can be written $x=c_{1}(y, \alpha)(0 \leqq \alpha \leqq 1)$ and since $\phi\left(\partial W_{1} \times\{1\}\right)=g c_{1}\left(\partial W_{1} \times\{1\}\right)$, we can write $\phi^{-1} g c_{1}(y, 1)=(u, 1)\left(u \in \partial W_{1}\right)$. Since $g=\phi$ on $c_{1}\left(\partial W_{1} \times\{1\}\right)$ by definition, $h$ is well defined.

Definition 2. Let $U_{g}$ be a region defined by Lemma 1 , then $U_{g}$ is an $h$-cobordism by Proposition 1 . We define $\tau\left(g_{\infty}\right)=\tau\left(U_{g}, g c_{1}\left(\partial W_{1} \times\{1\}\right)\right)$ $\in W h\left(\pi_{1}\left(\partial W_{1}\right)\right)$, Whitehead torsion near the boundary with respect to $g$.

Proposition 2. Let $W_{i}(i=1,2)$ be compact bounded manifolds and $f:$ Int $W_{1} \rightarrow$ Int $W_{2}$ be a PL homeomorphism with $\tau\left(f_{\infty}\right) \neq 0$, then there is no PL homeomorphism $\bar{f}: W_{1} \rightarrow W_{2}$ such that

$$
\bar{f}\left|W_{1}-c_{1}\left(\partial W_{1} \times[0,1]\right)=f\right| W_{1}-c_{1}\left(\partial W_{1} \times[0,1]\right) .
$$

(See also [5. chap. IX]).

Proof. If there is a $P L$ homeomorphism $\bar{f}$ as above,

$$
\begin{aligned}
\tau\left(f_{\infty}\right) & =\tau\left(U_{f}, f c_{1}\left(\partial W_{1} \times\{1\}\right)\right)=\tau\left(U_{f}, \bar{f} c_{1}\left(\partial W_{1} \times\{1\}\right)\right) \\
& =\tau\left(\partial W_{1} \times I, \partial W_{1} \times\{1\}\right)=0 .
\end{aligned}
$$

This is a contradiction.

THEOREM 1. Let $W^{n}(n \geqq 6)$ be a connected compact bounded PL $n$ manifold with a connected boundary $\partial W$. If $W h\left(\pi_{1}(\partial W)\right)=(0), \# \mathscr{A}(W)=1$. 
PRoof. For any $W_{1}, W_{2} \in \mathscr{A}(W)$, we will show $W_{1} \cong W_{2}$. Let $f$ : Int $W_{1} \rightarrow$ Int $W_{2}$ be a $P L$ homeomorphism and $U_{f}$ be a region bounded by $\partial W_{2}$ and $f c_{1}\left(\partial W_{1} \times\{1\}\right)$. Then $U_{f}$ is an $h$-cobordism by PROPOSITION 1 and $\tau\left(f_{\infty}\right)=\tau\left(U_{f}, f c_{1}\left(\partial W_{1} \times\{1\}\right)\right)=(-1)^{n-1} \tau\left(U_{f}, \partial W_{2}\right) \in W h\left(\pi_{1}\left(\partial W_{2}\right)\right) \cong W h\left(\pi_{1}(\partial W)\right)$ $=(0)\left(\left[4\right.\right.$, p. 394]). Hence by $s$-cobordism Theorem [6], $U_{f} \cong \partial W_{2} \times I \cong \partial W_{1}$ $\times I$ and so there is a $P L$ homeomorphism $\bar{f}: W_{1} \rightarrow W_{2}$ such that $\bar{f} \mid W_{1}-$ $c_{1}\left(\partial W_{1} \times[0,1]\right)=f \mid W_{1}-c_{1}\left(\partial W_{1} \times[0,1]\right)$ by LEMMA 1 .

PROPOSITION 3. Let $W_{i}^{n}(i=1,2)$ be compact bounded manifolds $(n \geqq 6)$ with connected boundaries $\partial W_{i}$ and Int $W_{1} \cong \operatorname{Int} W_{2}$. If $n$ is even and $\pi_{1}\left(\partial W_{1}\right)$ is finite abelian, $D_{\alpha} W_{1} \cong D W_{2}$ where $D W_{2}$ is the double of $W_{2}$ and $D_{a} W_{1}=W_{1} \cup W_{1}$ by some identification homeomorphism $\alpha: \partial W_{1} \rightarrow \partial W_{1}$. Furthermore if $\alpha$ is isotopic to identity, $D_{\alpha} W_{1} \cong D W_{1}$.

Proof. Let $W^{+}$and $W^{-}$be the copy of $W$ and $D W=W^{+} \cup W^{-}$. Let $f_{ \pm}$: Int $W_{1}^{ \pm} \rightarrow$ Int $W_{2}^{ \pm}$be homeomorphisms and $U_{f \pm}$ be regions bounded by $\partial W_{2}^{ \pm}$and $f_{ \pm} c_{1}^{ \pm}\left(\partial W_{1}^{ \pm} \times\{1\}\right)$ i.e. $\left(U_{f+} ; f_{+} c_{1}^{+}\left(\partial W_{1}^{+} \times\{1\}\right), \partial W_{2}^{+}\right)$and $U_{f_{-}}$: $\left.f_{-} c_{1}^{-}\left(\partial W_{1}^{-} \times\{1\}\right), \partial W_{2}^{-}\right)$. If $\tau=\tau\left(U_{f+}, f_{+} c_{1}^{+}\left(\partial W_{1}^{+} \times\{1\}\right)\right), \tau\left(U_{f-}, \partial W_{2}^{-}\right)=$ $(-1)^{n-1} \bar{\tau}$ [4. p. 394] and so $\tau\left(U_{f+} \bigcup_{\partial W_{2}} U_{f-}, f_{+} c_{1}^{+}\left(\partial W_{1}^{+} \times\{1\}\right)\right)=\tau+(-1)^{n-1} \bar{\tau} \epsilon$ $W h\left(\pi_{1}\left(\partial W_{1}\right)\right)$ [4. Th. 3. 2] where $\left(U_{f+} \cup U_{f^{-}} ; f_{+} c_{1}^{+}\left(\partial W_{1}^{+} \times\{1\}\right)\right), f_{-} c_{1}^{-}\left(\partial W_{1}^{-} \times\right.$ $\{1\}))$ is an $h$-cobordism obtained from $U_{f+} \cup U_{f-}$ by $\partial W_{2}$ identiffied. Since $\pi_{1}(\partial W)$ is finite abelian, $\tau=\bar{\tau}[4]$ and so $\tau\left(U_{f+} \cup U_{f-}, f_{+} c_{1}^{+}\left(\partial W_{1}^{+} \times\{1\}\right)\right)=0$ if $n$ is even. Hence $U_{f+} \cup_{\partial W_{2}} U_{f-} \cong \partial W_{1} \times I$ by $s$-cobordism Theorem. Similarly if

$$
\begin{aligned}
& U_{f-}^{\prime}=\left(U_{f-}^{\prime}: \partial W_{2}^{-}, f_{-} c_{1}^{-}\left(\partial W_{1}^{-} \times\left\{\frac{1}{2}\right\}\right)\right) \text { and } \\
& U_{f-}^{\prime \prime}=\left(U_{f^{\prime}}^{\prime \prime}: f_{-} c_{1}^{-}\left(\partial W_{1}^{-} \times\left\{\frac{1}{2}\right\}\right), f_{-} c_{1}^{-}\left(\partial W_{1}^{-} \times\{1\}\right)\right), \\
& U_{f+} \cup U_{f-}^{\prime} \cong \partial W_{1} \times I \text { and } U_{f^{\prime}}^{\prime \prime} \cong \partial W_{1} \times I .
\end{aligned}
$$

Let $\phi_{1}: \partial W_{1} \times I \rightarrow U_{f+} \cup U_{f-}^{\prime}$ be a homeomorphism such that $\phi_{1}\left(\partial W_{1} \times\{0\}\right)=$ $f_{+} c_{1}\left(\partial W_{1}^{+} \times\{1\}\right)$

$$
\phi_{1}\left(\partial W_{1} \times\{1\}\right)=f_{-} c_{1}^{-}\left(\partial W_{1}^{-} \times\left\{\frac{1}{2}\right\}\right)
$$

and let

$$
\phi_{2}:\left(\partial W_{1}^{+} \times[0,1]\right) \cup\left(\partial W_{1}^{-} \times\left[0, \frac{1}{2}\right]\right) \rightarrow \partial W_{1} \times I
$$

be a homeomorphism defined by 


$$
\begin{aligned}
& \phi_{2}\left(y^{+}, 1-t\right)=\left(z^{+}\left(y^{+}\right), \frac{t}{2}\right), 0 \leqq t \leqq 1 \\
& \phi_{2}\left(y^{-}, t\right)=\left(z^{+}\left(y^{+}\right), \frac{1}{2}+t\right), 0 \leqq t \leqq \frac{1}{2}
\end{aligned}
$$

where

$$
y^{+} \in \partial W_{1}^{+}, y^{-} \in \partial W_{1}^{-} \quad \text { and } \phi_{1}^{-1} f_{+} c_{1}^{+}\left(y^{+}, 1\right)=\left(z^{+}\left(y^{+}\right), 0\right)
$$

And we define a homeomorphism $\gamma: D W_{1} \rightarrow D W_{1}$ by

$$
\begin{aligned}
& \gamma \mid\left(W_{1}^{+}-c_{1}^{+}\left(\partial W_{1}^{+} \times[0,1)\right)\right) \cup\left(W_{1}^{-}-c_{1}^{-}\left(\partial W_{1}^{-} \times[0,1)\right)\right)=i d . \\
& \gamma c_{1}^{+}\left(y^{+}, t\right)=c_{1}^{+}\left(y^{+}, \frac{1}{2}(3 t-1)\right) \frac{1}{3} \leqq t \leqq 1 \\
& \gamma c_{1}^{+}\left(y^{+}, t\right)=c_{1}^{-}\left(y^{-}, \frac{1}{2}(1-3 t)\right) 0 \leqq t \leqq \frac{1}{3} \\
& \gamma c_{1}^{-}\left(y^{-}, t\right)=c_{1}^{-}\left(y^{-}, \frac{1}{2}(1+t)\right) \quad 0 \leqq t \leqq 1,
\end{aligned}
$$

and let $\beta$ : $c_{1}^{-}\left(\partial W_{1}^{-} \times\left\{\frac{1}{2}\right\}\right) \rightarrow c_{1}^{-}\left(\partial W_{1}^{-} \times\left\{\frac{1}{2}\right\}\right)$ be a homeomorphism defined by

$$
\beta c_{1}^{-}\left(y^{-}, \frac{1}{2}\right)=\left(f_{-}\right)^{-1} \phi_{1} \phi_{2}\left(y^{-}, \frac{1}{2}\right)
$$

We define a homeomorphism $\alpha: \partial W_{1} \rightarrow \partial W_{1}$ by

$$
\alpha=\gamma^{-1} \beta\left(\gamma \mid c_{1}^{+}\left(\partial W_{1} \times\{0\}\right)\right) .
$$

Then there is a well-defined homeomorphism $h: D_{\alpha} W_{1} \rightarrow D W_{2}$ defined by

$$
h(x)= \begin{cases}f_{+}\left(x^{+}\right) & x^{+} \in W_{1}^{+}-c_{1}^{+}\left(\partial W_{1}^{+} \times[0,1)\right) \\ \phi_{1} \phi_{2}\left(c_{1}^{-}\right)^{-1} \gamma\left(x^{+}\right) & x^{+} \in c_{1}^{+}\left(\partial W_{1}^{+} \times\left[0, \frac{1}{3}\right]\right) \\ \phi_{1} \phi_{2}\left(c_{1}^{+}\right)^{-1} \gamma\left(x^{+}\right) & x^{+} \in c_{1}^{+}\left(\partial W_{1}^{+} \times\left[\frac{1}{3}, 1\right]\right) \\ f_{-} \gamma\left(x^{-}\right) & x^{-} \in W_{1}^{-}\end{cases}
$$

Next we will show $D_{\alpha} W_{1} \cong D W_{1}$ if $\alpha$ is isotopic to identity. Since $\alpha \approx i d$, there is a level preserving homeomorphism $H: \partial W_{1} \times I \rightarrow \partial W_{1} \times I$ such that $H_{0}=\alpha$ and $H_{1}=i d$. Let $c_{1}: \partial W_{1} \times I \rightarrow W_{1}$ be a collar (embedding) such that $c_{1}(y, 0)=y \in \partial W_{1}$ and $c_{1}^{\alpha}: \partial W_{1} \times I \rightarrow W_{1}$ be $c_{1}^{\alpha}(y, t)=c_{1} H(y, t)$. Then we define 
a homeomorphism

$$
F: \quad D W_{1} \rightarrow D_{a} W_{1}
$$

by

$$
\begin{array}{ll}
F\left(x^{+}\right)=x^{+} & x^{+} \in W_{1}^{+} \\
F\left(x^{-}\right)=x^{-} & x^{-} \in W_{1}^{-}-c_{1}^{-}\left(\partial W_{1}^{-} \times[0,1)\right) \\
F\left(x^{-}\right)=F\left(c_{1}^{-}\left(y^{-}, t\right)\right)=c_{1}^{\alpha}\left(y^{-}, t\right) & x^{-} \in c_{1}^{-}\left(\partial W_{1}^{-} \times[0,1]\right) .
\end{array}
$$

Hence $D W_{1} \cong D_{\alpha} W_{1}$.

Definition 3. Let $W^{n}(n \geqq 6)$ be a compact bounded $n$-manifold. Then $I[W, \partial W]$ is the inertia group defined by [2] i. e.

$$
I[W, \partial W]=\left\{\tau \in W h\left(\pi_{1}(\partial W)\right) \mid(W, \partial W) \circ \tau=(W, \partial W)\right\}
$$

where $(W, \partial W) \circ \tau=\left(W \cup U, \partial W^{\prime}\right)$ and $\left(U ; \partial W, \partial W^{\prime}\right)$ is an $h$-cobordism with $\tau(U, \partial W)=\tau$. Similarly if $M$ is a closed $n$-manifold $(n \geqq 5), I[M]=\{\tau \epsilon$ $\left.W h\left(\pi_{1}(M)\right) \mid M \circ \tau=M\right\}$ where $M \circ \tau=M^{\prime}$ and $\left(U ; M, M^{\prime}\right)$ is an $h$-cobordism with $\tau(U, M)=\tau$.

Let $\langle W, \partial W\rangle$ be a set of manifolds $\left(W^{\prime}, \partial W\right)$ such that $(W, \partial W) \circ \tau=$ $\left(W^{\prime}, \partial W\right), \tau \in W h\left(\pi_{1}(\partial W)\right)$ and

$$
\begin{array}{r}
\tilde{I}[W, \partial W]=\left\{\tau \in W h\left(\pi_{1}(\partial W)\right) \mid\left(W^{\prime}, \partial W\right) \circ \tau=\left(W^{\prime}, \partial W\right)\right. \\
\text { for any } \left.\left(W^{\prime}, \partial W\right) \in\langle(W, \partial W)\rangle\right\} .
\end{array}
$$

Then the following Lemma is obvious by definition.

LEMMA 2. \#Ĩ $[W, \partial W] \leqq \# I[W, \partial W] \leqq \# I[\partial W] \leqq \# W h\left(\pi_{1}(\partial W)\right)$. Using s-cobordism Theorem we obtain the following.

PROPOSITION 4. Let $W^{n}$ be a compact $n$-manifold $(n \geqq 6)$ with $\partial W=$ $M_{1} \cup M_{2}$ where $M_{i}(i=1,2)$ are connected. If $\left(W ; M_{1}, M_{2}\right)$ is an h-cobordism, $\# \mathscr{A}(W)=\# I\left[M_{1}\right]=\# I\left[M_{2}\right]$.

Proof. CASE 1. $M_{1} \cong M_{2}$. We define a map $\alpha: I\left[M_{1}\right] \rightarrow \mathscr{A}(W)$ by $\alpha(\tau)=\widetilde{W}$ where $\widetilde{W}$ is an $h$-cobordism from $M_{1}$ with $\tau\left(\widetilde{W}, M_{1}\right)=\tau$. Then $\widetilde{W}$ is uniquely determined by $\tau$ up to $P L$ homeomorphism class [4. Th. 11. 3] and $\widetilde{W} \in \mathscr{A}(W)$ because Int $\widetilde{W} \cong M_{1} \times R \cong$ Int $W$ and $\partial \widetilde{W}=M_{1} \cup M_{1}$ since $\tau \in I\left[M_{1}\right]$. And if $\tau_{1} \neq \tau_{2} \in I\left[M_{1}\right], \widetilde{W}_{1} \neq \widetilde{W}_{2}$ by [4. Th. 11. 3] where $\alpha\left(\tau_{i}\right)=\widetilde{W}_{i}$ $(i=1,2)$. So $\alpha$ is injective, clearly for any $\widetilde{W} \in \mathscr{A}(W), \tau \in I\left[M_{1}\right]$ where $\tau=\tau\left(\widetilde{W}, M_{1}\right)$. Hence $\alpha$ is onto.

CASE 2. $M_{1} \not M_{2}$. Let $\tau=\tau\left(W, M_{1}\right)$ fix. And we define a map $\alpha$ : 
$I\left[M_{1}\right] \rightarrow \mathscr{A}(W)$ by $\alpha(\omega)=U \bigcup_{M_{1}} W$ where $U$ is an $h$-cobordism from $M_{1}$ with $\tau\left(U, M_{1}\right)=\omega$. Then $\partial\left(U \cup_{M_{1}}^{M_{1}} W\right)=M_{1} \cup M_{2}$ and Int $(U \cup W) \cong M_{1} \times R \cong \operatorname{Int} W$ by [1. vol. II]. So $U \bigcup_{M_{1}} W \in \mathscr{A}(W)$. If $\tau_{1} \neq \tau_{2} \in I\left[M_{1}\right], U_{1} \bigcup_{M_{1}} W \neq U_{2} \bigcup_{M_{1}} W$ by [4. 11. 3]. Hence $\alpha$ is injective. Now we will show $\alpha$ surjective. For any $\widetilde{W} \in \mathscr{A}(W)$, since $\widetilde{W}-M_{1} \cong W-M_{1} \cong M_{2} \times[0, \infty)$. there is a $P L$ homeomorphism $f: W-M_{1} \rightarrow \widetilde{W}-M_{1}$. Let $U_{f}$ be a bounded region by $M_{1}$ and $f c_{1}\left(M_{1} \times\{1\}\right)$ in $\widetilde{W}$ where $c_{1}: M_{1} \times I \rightarrow W$ is a boundary collar and let $\omega_{f}=\tau\left(U_{f}, M_{1}\right)$. Then $\omega_{f} \in I\left[M_{1}\right]$. Now $\omega_{f}$ does

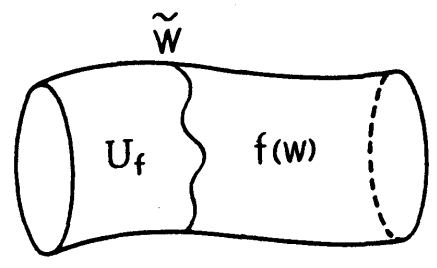

Fig. 2. not depend on $f$ because if $g: W-M_{1} \rightarrow \widetilde{W}-M_{1}$ is another PL homeomorphism,

$$
\begin{aligned}
\tau\left(U_{g},\right. & \left.M_{1}\right)+\tau\left(g(W), g\left(M_{1}\right)\right) \\
& =\omega_{g}+\tau=\tau\left(\widetilde{W}, M_{1}\right) \\
& =\tau\left(U_{f}, M_{1}\right)+\tau\left(f\left(W, f\left(M_{1}\right)\right)=\omega_{f}+\tau\right.
\end{aligned}
$$

and so $\omega_{g}=\omega_{f}$. Hence $\alpha$ is onto and $\# I\left[M_{1}\right]=\# \mathscr{A}(W)$. Similarly $\# I\left[M_{2}\right]$ $=\# \mathscr{A}(W)$.

Corollary. If $W$ is the same as Proposition 4 , \#A $(W) \geqq$ $\# d^{n}\left(W h\left(\pi_{1}\left(M_{1}\right)\right)\right)$ where $d_{n}: W h\left(\pi_{1}\left(M_{1}\right)\right) \rightarrow W h\left(\pi_{1}\left(M_{1}\right)\right)$ is an endomorphism defined by $d_{n}(\tau)=\tau+(-1)^{n-1} \bar{\tau}$.

Proof follows by the fact that $d_{n}\left(W h\left(\pi_{1}\left(M_{1}\right)\right)\right.$ is a subgroup of $I\left[M_{1}\right]$.

Definition 4 . Let $W$ be a $P L$ manifold and $K$ be a subpolyhedron. We say $K$ homotopy spine of $W$ if the polyhedral pair $(W, K)$ is an abstract $h$-neighborhood i.e. $W, K$ satisfy the following conditions (see [2]):

(1) $K \subset$ Int $W$

(2) for some regular neighborhood $N$ of $K$ in $W,(\overline{W-N} ; \partial W, \partial N)$ is an $h$-cobordism.

Definition 5. If $\tau \in I[W, \partial W]$, by the definition there exist an $h$ cobordism $(U ; \partial W, \partial W)$ with $\tau(U, \partial W)=\tau$ and $P L$ homeomorphism

$h: W \cup U \rightarrow W$. We define $I[W, \partial W ; i d]=\{\tau \in I[W, \partial W]|h| K$ is homotopic to inclusion $i: K \rightarrow W$ for some homotopy spine $K$ of $W\}$.

LemMA 3. Let $W^{n}$ be a compact bounded n-manifold $(n \geqq 6)$ with a connected boundary. If $i_{*}: W h\left(\pi_{1}(\partial W)\right) \rightarrow W h\left(\pi_{1}(W)\right)$ is a monomorphism, $I[W, \partial W ; i d]=0$.

Proof. Let $\tau \in I[W, \partial W ; i d],(U ; \partial W, \partial W)$ be an $h$-cobordism with 
$\tau(U, \partial W)=\tau$ and $h: W \cup U \rightarrow W$ be a homeomorphism such that $h \mid K \simeq i$ for some homotopy spine $K$ of $W$. Then by $[4: 7.6,7.7]$

$$
\tau(W, h(K))=\tau(h)=\tau(i)=\tau(W, K) \in W h\left(\pi_{1}(W)\right) .
$$

And since $h$ is a homeomorphism,

$$
\tau(W, h(K))=i_{*} \tau(U, \partial W)+\tau(W, K) .
$$

Hence $i_{* \tau}(U, \partial W)=i_{*} \tau=0$ and $\tau=0$.

THEOREM 2. If $W^{n}$ is a compact bounded n-manifold $(n \geqq 6)$ with a connected boundary, then $\# \mathscr{A}(W)=\#\langle(W, \partial W)\rangle \leqq \# I[\partial W]$. Furthermore if $\operatorname{ker}\left(i_{*}: W h\left(\pi_{1}(\partial W)\right) \rightarrow W h\left(\pi_{1}(W)\right)\right)=0$ and $I[W, \partial W: i d]=I[W, \partial W], \# \mathscr{A}(W)$ $=\# I[\partial W]$.

Proof. Let $W_{1} \in \mathscr{A}(W)$ such that $W_{1} \neq W$ and let $f:$ Int $W_{1} \rightarrow$ Int $W$ be a homeomorphism. Let $U_{f}$ be a region bounded by $\partial W$ and $f c_{1}\left(\partial W_{1}\right.$ $\times\{1\})$. Then $\left(U_{f} ; \partial W, f_{c_{1}}\left(\partial W_{1} \times\{1\}\right)\right)$ is an $h$-cobordism by Proposition 1 and $\tau\left(U_{f}, f c_{1}\left(\partial W_{1} \times\{1\}\right)\right) \neq 0$ in $I\left[\partial W_{1}\right]=I[\partial W]$ for any $f$ because $\partial W_{1} \cong \partial W$ and $W_{1} \neq W$. Similarly if $W_{1}, W_{2} \in \mathscr{A}(W)$ such that $W_{1} \neq W \neq W_{2}, W_{1} \neq W_{2}$, then $\tau\left(U_{f}, f c_{1}\left(\partial W_{1} \times\{1\}\right)\right) \neq \tau\left(U_{g}, g c_{2}\left(\partial W_{2} \times\{1\}\right)\right)$ where $g:$ Int $W_{2} \rightarrow$ Int $W$ is a homeomorphism. So $\# \mathscr{A}(W) \leqq \# I[\partial W]$.

Since $\operatorname{Int}\left(W \cup \underset{\partial W}{\cup} \cong\right.$ Int $W$ where $U$ is an $h$-cobordism from $\partial W, W^{\prime} \in$ $\mathscr{A}(W)$ if $W^{\prime} \in\langle(W, \partial W)\rangle$. So $\#\langle W, \partial W\rangle \leqq \# \mathscr{A}(W)$. And if $W_{1}, W_{2} \in$ $\mathscr{A}(W)$, Int $W_{1} \cong$ Int $W_{2}$ so $W_{1}$ is boundary $h$-cobordant to $W_{2}$ by ProposiTION 1 and $\partial W_{1} \cong \partial W_{2}$. Hence $W_{1}, W_{2} \in\langle W, \partial W\rangle$ and $\mathscr{A}(W)=\langle(W, \partial W)\rangle$. Since $\# \mathscr{A}(W)=\#(I[\partial W] / I[W, \partial W])$, by Lemma 3

$$
\# \mathscr{A}(W)=\# I[\partial W] \text { if } \operatorname{ker} i_{*}=0
$$

and $I[W, \partial W: i d]=I[W, \partial W]$.

THEOREM 3. Let $M^{n}$ be a closed n-manifold $(n \geqq 5)$ and let $G=\{\tau \in$ $W h\left(\pi_{1}\left(M \times S^{1}\right)\right) \mid$ if $A=\left(a_{i j}\right) \in G L\left(p, Z_{1}\left(M \times S^{1}\right)\right)$ is a representative of $\tau, a_{i j} \in$ $\left.Z_{\pi_{1}}(M) \otimes\{1\}\right\}$. Then there is a homomorphism $\phi$ of $I[M]$ onto $G /(I[M \times$ $\left.\left.S^{1}\right] \cap G\right)$ with $\operatorname{ker} \phi \supset\left\{\omega \in I[M] \mid \omega+(-1)^{n} \bar{\omega}=0\right\}$.

COROLlARY. If $M^{n}$ is a closed $n$-manifold ( $n \geqq 5$ and odd) with $\pi_{1}(M)$ = finite abelian, $G \subset I\left[M \times S^{1}\right]$.

Proof. If $M$ satisfies all conditions, for any $\tau \in W h\left(\pi_{1}(M)\right) \tau+(-1)^{n} \bar{\tau}$ $=0$ by [4]. So $\left\{\tau \in I[M] \mid \tau+(-1)^{n} \bar{\tau}=0\right\}=I[M] \subset$ ker $\phi$. Hence $G /\left(I\left[M \times S^{1}\right]\right.$ $\cap G)=0$ by THEOREM 3 .

Proof of Theorem 3. Let $U$ be an $h$-cobordism from $M$ to itself. 
Then Int $U \cong M \times R$ and so $U$ is boundary $h$-cobordant to $M \times I$ by PROPOSition 1 i.e. $(M \times I ; M, M) \cong\left(V_{1} \cup \cup \cup \bigcup_{M} ; M, M\right)$ for some $h$-cobordisms $\left(V_{1} ; M, M\right),\left(V_{2} ; M, M\right)$. Let $\left(\widetilde{W} ; V_{1} \cup U \cup V_{2}, M \times I\right)$ be a trivial $h$-cobordism. Then $(W ; U, M \times I)$ is an $h$-cobordism between $U$ and $M \times I$ where

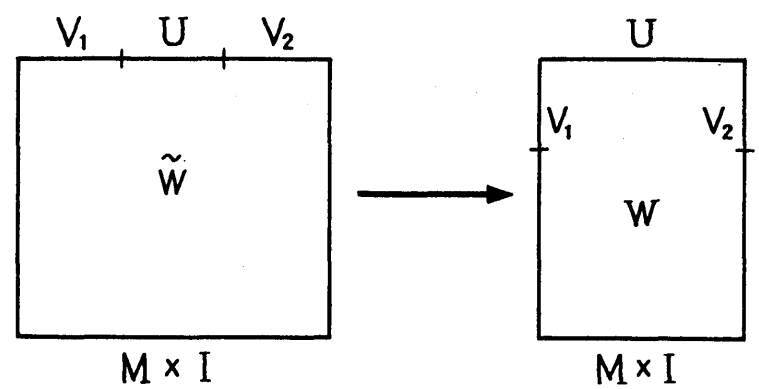

Fig. 3.

$\partial W \cong \partial \widetilde{W}$. Let $\omega_{1}=\tau\left(V_{1}, M\right), \omega_{2}=\tau\left(V_{2}, M\right)$ and let $\left(V_{1}^{\prime} ; M, M\right),\left(V_{2}^{\prime} ; M, M\right)$ be $h$-cobordisms with $\tau\left(V_{1}^{\prime}, M\right)=-\omega_{1}, \tau\left(V_{2}^{\prime}, M\right)=-\omega_{2}$. Then $V_{1} \cup V_{1}^{\prime}$ and $V_{2} \cup V_{2}^{\prime}$ are both trivial $h$-cobordism and so there are trivial $h$-cobordisms $W_{1}, W_{2}$ between $V_{1} \cup V_{1}^{\prime}, V_{2} \cup V_{2}^{\prime}$ and $M \times I$. Therefore $V_{1}^{\prime} \cup U \cup V_{2}^{\prime}$ is $h$ cobordant to $M \times I$ by the $h$-cobordism $W_{1} \cup W \cup W_{2}$ such that

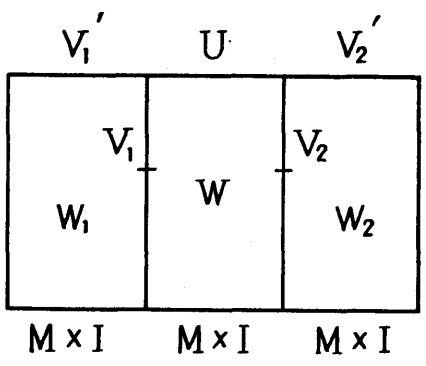

Fig. 4.

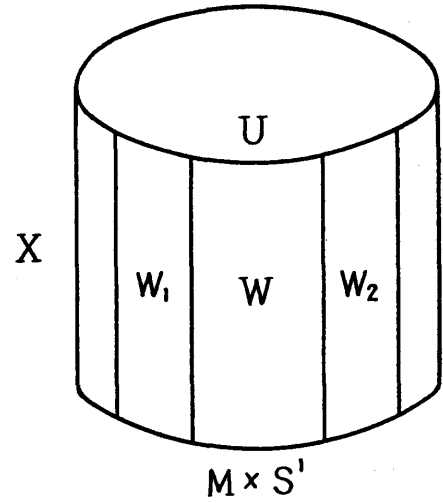

Fig. 5 .

$\partial\left(V_{1}^{\prime} \cup U \cup V_{2}^{\prime}\right) \cong M \times S^{0}$ is trivially $h$-cobordant to $M \times S^{0}$ by the same $W_{1} \cup$ $W \cup W_{2}$. Let $X=(M \times I \times J) \cup\left(W_{1} \cup W \cup W_{2}\right)$ by identifying $M \times(0) \times(t) \sim$ $M \times(t) \subset$ "free" part of $\partial W_{1}, M \times\{1\} \times\{t\} \sim M \times\{t\} \subset$ "free" part of $\partial W_{2}$. Then $X$ is an $h$-cobordism from $M \times S^{1}$. So there is a map

$$
\bar{\phi}: I[M] \rightarrow W h\left(\pi_{1}\left(M \times S^{1}\right)\right)
$$

defined by

$$
\bar{\phi}(\tau)=\tau\left(X, M \times S^{1}\right) \quad \text { where } \tau=\tau(U, M) .
$$

Let $(\widetilde{U} ; M, M)$ be an $h$-cobordism with $\tau(\widetilde{U} ; M)=\tilde{\tau} \neq \tau \in I[M]$. Then 
$U \not \widetilde{U}$. And let $\widetilde{V}_{1}^{\prime}, \widetilde{V}_{2}^{\prime}$ be the corresponding $h$-cobordism for $\widetilde{U}$ as above. If $\left(\widetilde{V}_{1}^{\prime} \cup \widetilde{U} \cup \widetilde{V}_{2}^{\prime}\right) \cup M \times I \times\{1\} \neq\left(V_{1}^{\prime} \cup U \cup V_{2}^{\prime}\right) \cup(M \times I \times\{1\}), \tau\left(\widetilde{X}, M \times S^{1}\right) \neq \tau(X$, $\left.M \times S^{1}\right)$. But if $\left(\widetilde{V}_{1}^{\prime} \cup U \cup \widetilde{V}_{2}^{\prime}\right) \cup(M \times I \times\{1\}) \cong\left(V_{1}^{\prime} \cup U \cup V_{2}^{\prime}\right) \cup(M \times I \times\{1\})$, I don't know whether $\tau\left(\widetilde{X}, M \times S^{1}\right)=\tau\left(X, M \times S^{1}\right)$ or not. So we define a map $\phi: I[M] \rightarrow W h\left(\pi_{1}\left(M \times S^{1}\right)\right) / I\left[M \times S^{1}\right]$ by $\phi(\tau)=\left[\tau\left(X, M \times S^{1}\right)\right]$. Then $\phi$ is well defined. And since $M \times I \times J \cup N\left(M \times 3 I, W_{1} \cup W \cup W_{2}\right) \subset X$ is homeomorphic to $M \times S^{1} \times J$ where $M \times 3 I=(M \times I) \cup(M \times I) \cup(M \times I) \cup \partial\left(W_{1} \cup W\right.$ $\left.\cup W_{2}\right)$ and $N\left(M \times 3 I, W_{1} \cup W \cup W_{2}\right)$ is a regular neighborhood of $M \times 3 I$ in $W_{1} \cup W \cup W_{2}$, we may consider that $X$ is constructed from $M \times S^{1} \times J$ by attaching handles on $M \times I \times\{1\} \subset M \times S^{1} \times\{1\}$. So if $A=\left(a_{i j}\right) \in G L(p$, $\left.Z \pi_{1}\left(M \times S^{1}\right)\right)$ is a representation of $\tau\left(X, M \times S^{1}\right), a_{i j} \in Z_{\pi_{1}}(M) \otimes\{1\}$. Hence Im $\phi \subset G /\left(I\left[M \times S^{1}\right] \cap G\right)$. Now we will show $\phi$ surject!ve. Let $[\omega]$ be an element of $G /\left(I\left[M \times S^{1}\right] \cap G\right)$ such that $\omega=\tau\left(X, M \times S^{1}\right)$. Since a representative $A=\left(a_{i j}\right)$ of $\omega$ in $G L\left(p, Z \pi_{1}\left(M \times S^{1}\right)\right)$ has a form $a_{i j} \in Z \pi_{1}(M) \otimes\{1\}$, we may assume

$$
\begin{aligned}
X & =\left(M \times S^{1} \times J\right) \cup\{\text { handles }\} \\
& =\left(M \times I_{1} \times J\right) \cup\left(M \times I_{2} \times J\right) \cup\{\text { handles }\}
\end{aligned}
$$

so that all handles do not attach one of $M \times I \times J_{s}^{\prime}$, say $M \times I_{1} \times J$ [4]. Let $U=\partial X-\left(M \times S^{1} \times\{0\} \cup M \times I_{1} \times\{1\}\right)$. Then $U$ is an $h$-cobordism between $M$. So there is an element $\tau(U, M) \in I[M]$ such that $\phi(\tau(U, M))=[\omega]$. Now let $\omega$ be an element of $I[M]$ such that $\omega+(-1)^{n} \bar{\omega}=0$. Then $U \cup \bar{U}$ $\cong M \times I$ where $\tau(U, M)=\omega$ and $\tau(\bar{U}, M)=(-1)^{n} \overline{\omega^{\prime}}$. So $((U \cup \bar{U}) \times J) \cup(M \times \partial I \times J$ $\times I \times J) \cong M \times S^{1} \times J$. Hence $\phi(\omega)=0$ and so $\operatorname{ker} \phi \supset\left\{\omega \in I[M] \mid \omega+(-1)^{n} \bar{\omega}\right.$ $=0\}$.

Now, suppose $R$ and $R^{\prime}$ are rings which are also algebras over the commutative ring $A$, and let $C$ be a free $R$-complex with a preferred basis, and $C^{\prime}$ a free $R^{\prime}$ complex with a preferred basis. Then $C \otimes_{A} C^{\prime}$ is a free $R \otimes{ }_{A} R^{\prime}$ compelx with a preferred basis.

We obtain the following proposition by [4. §3].

Proposition 5. Let $C$ be a free $R$-complex with a preferred basis, and $C^{\prime}$ a free $R^{\prime}$-complex with a preferred basis. Then if $H_{*}(C)$ and $H_{*}\left(C^{\prime}\right)$ are both free, so is $H_{*}\left(C \otimes_{A} C^{\prime}\right)$ and $\tau\left(C \otimes{ }_{A} C^{\prime}\right)=\tau(C \otimes B)+\tau\left(C \otimes B^{\prime}\right)$ $+\tau(\mathscr{H})$ where $B$ and $B^{\prime}$ are $R^{\prime}$-complexes such that

$$
\begin{aligned}
& B: \quad 0 \rightarrow C_{n}^{\prime} \rightarrow C_{n-1}^{\prime} \rightarrow \cdots \rightarrow C_{p+1}^{\prime} \rightarrow 0 \\
& B^{\prime}: \quad 0 \rightarrow C_{p}^{\prime} \rightarrow C_{p-1}^{\prime} \rightarrow \cdots \rightarrow C_{0}^{\prime} \rightarrow 0
\end{aligned}
$$

for any $p$ when 


$$
0 \rightarrow C_{n}^{\prime} \rightarrow C_{n-1}^{\prime} \rightarrow \cdots \rightarrow C_{p}^{\prime} \rightarrow C_{p-1}^{\prime} \rightarrow \cdots \rightarrow C_{0}^{\prime} \rightarrow 0
$$

is a chain complex $C^{\prime}$ and where $\mathscr{A}$ is an $R \otimes_{A} R^{\prime}$ complex $H_{m}(C \otimes B) \rightarrow$ $H_{m}\left(C \otimes C^{\prime}\right) \rightarrow H_{m}\left(C \otimes B^{\prime}\right) \rightarrow H_{m-1}(C \otimes B) \rightarrow \cdots \rightarrow H_{0}\left(C \otimes B^{\prime}\right) \rightarrow 0$ induced by the short exact sequence

$$
0 \rightarrow C \otimes B \rightarrow C \otimes C^{\prime} \rightarrow C \otimes B^{\prime} \rightarrow 0 .
$$

Corollary. If $M$ is a manifold such that $H_{*}(\widetilde{M})$ is free $Z_{\pi_{1}}(M)$ module where $\widetilde{M}$ is a universal covering space, $\tau\left(M \times S^{1}\right)\left(\stackrel{\operatorname{def}}{=} \tau\left(c\left(\widetilde{M \times S^{1}}\right)\right)\right) \in$ $W h\left(\pi_{1}\left(M \times S^{1}\right)\right)$ is equal to $\tau(c(\widetilde{\boldsymbol{M}}))+\tau(\mathscr{H})$, where $\mathscr{A}$ is a $Z \pi_{1}(M) \otimes_{z} Z \pi_{1}\left(S^{1}\right)$ complex

$$
\begin{aligned}
H_{m}\left(c(\widetilde{\boldsymbol{M}}) \otimes c_{1}\left(\widetilde{\boldsymbol{S}}^{1}\right)\right) & \rightarrow H_{m}\left(c(\widetilde{\boldsymbol{M}}) \otimes c\left(\tilde{\boldsymbol{S}}^{1}\right)\right) \rightarrow H_{m}\left(c(\widetilde{\boldsymbol{M}}) \otimes c_{0}\left(\widetilde{S}^{1}\right)\right) \\
& \rightarrow \cdots \rightarrow H_{0}\left(c(\widetilde{\boldsymbol{M}}) \otimes c_{0}\left(\widetilde{\boldsymbol{S}}^{1}\right)\right) \rightarrow 0 .
\end{aligned}
$$

Proof. By Proposition 5

$$
\begin{aligned}
\tau\left(c(\widetilde{\boldsymbol{M}}) \otimes c\left(\widetilde{S}^{1}\right)\right) \\
\quad=\tau\left(c(\widetilde{\boldsymbol{M}}) \otimes c_{1}\left(S^{1}\right)\right)+\tau\left(c(\widetilde{\boldsymbol{M}}) \otimes c_{0}\left(\widetilde{S}^{1}\right)\right)+\tau(\mathscr{H}) \\
\quad=\chi\left(c_{1}\left(\widetilde{S}^{1}\right)\right) \tau(c(\widetilde{\boldsymbol{M}}))+\chi\left(c_{0}\left(\widetilde{S}^{1}\right)\right) \tau(c(\widetilde{\boldsymbol{M}}))+\tau(\mathscr{H}) \\
\quad=\chi\left(c\left(\widetilde{S}^{1}\right)\right) \tau(c(\widetilde{\boldsymbol{M}}))+\tau(\mathscr{A})
\end{aligned}
$$

where $\chi\left(c\left(\widetilde{S}^{1}\right)\right)$ is the euler characteristic as $Z_{\pi_{1}}\left(S^{1}\right)$-complex. Since $\widetilde{S}^{1}=\widetilde{R}^{1}$, $\chi\left(c\left(\widetilde{S}^{1}\right)\right)=1$. Hence $\tau\left(c(\widetilde{\boldsymbol{M}}) \otimes c\left(\widetilde{S}^{1}\right)\right)=\tau(c(\widetilde{\boldsymbol{M}}))+\tau(\mathscr{H})$. And since $\widetilde{M \times S}=$ $\widetilde{M} \times \widehat{S}^{1}$, we obtain the result.

Hokkaido University

\section{References}

[1] L. C. Glaser : Geometrical combinatorial topology Vol. II. van Nostrand Reinhold Math. Studies.

[2] M. KAto: Geometrical operation of Whitehead groups, J. Math. Soc. Japan, Vol. 21 (4) 1969 pp 523-542.

[3] Kyung Whan KWUN and R. H. Szczarba: Product and sum theorems for Whitehead torsion, Ann. Math., Vol. 82 (1965)pp 183-190.

[ 4] J. MilnoR: Whitehead torsion, Bull. Amer. Math. Soc., Vol. 72 (3) 1966 pp 358426.

[5] L. C. SiebenmanN: The obstruction to finding a boundary for an open mani- 
fold of dimension greater than five. ph. D. Thesis, Princeton Univ. (1965).

[6] J. R. Stallings: Lectures on polyhedral topology, Tata Inst. Fund. Research, Bombay (1968).

(Received February 9, 1974)

(Revised March 25, 1974) 